\title{
Diretrizes para o Design de Visualização da Informação: Estendendo a Linguagem Cidadã
}

\author{
Rodrigo Oliveira ${ }^{1}$, Cláudia Cappelli ${ }^{2}$, Jonice Oliveira ${ }^{1}$ \\ ${ }^{1}$ Programa de Pós-Graduação em Informática (PPGI) da Universidade Federal do Rio de \\ Janeiro (UFRJ), Rio de Janeiro, RJ, Brasil \\ ${ }^{2}$ Departamento de Ciência da Computação e Informática da Universidade do Estado do \\ Rio de Janeiro (UERJ), Rio de Janeiro, RJ, Brasil \\ rodrigo.oliveira@ppgi.ufrj.br, claudia.cappelli@gmail.com, \\ jonice@dcc.ufrj.br
}

\begin{abstract}
Citizen Language is a technique that seeks to improve the understanding of texts through a set of practices. However, there are no specific guidelines to improve understanding of data. We publish here a proposal to complement the practices of Citizen Language with specific elements for this purpose. A set of guidelines selected with the methodological rigor of a literature review in the area of information visualization. The objective is to support the non-specialist developer in the production of more efficient views, whether for reports or public portals of published data and thus contributing to the citizen's understanding.
\end{abstract}

Resumo. A Linguagem Cidadã é uma técnica que busca melhorar o entendimento de textos através de um conjunto de práticas. Contudo, não há diretrizes específicas para melhorar entendimento de dados. Divulgamos aqui uma proposta de complementação das práticas da Linguagem Cidadã com elementos específicos para este fim. Um conjunto de diretrizes selecionadas com o rigor metodológico de uma revisão da literatura da área de visualização de informações. $O$ objetivo é apoiar o desenvolvedor não especialista na produção de visualizações mais eficientes, sejam para relatórios ou portais públicos de dados abertos e assim contribuir para o entendimento do cidadão.

\section{Introdução}

Em virtude do aumento da adesão aos conceitos de transparência pública há um crescente surgimento de portais de serviços e dados com informações geradas continuamente, sejam elas, relativas a finanças, saúde, desenvolvimento urbano, etc. (Barcellos et al., 2017). A Lei de acesso à informação (Lei Federal $\mathrm{n}^{\circ} 12.527$ ) criada em 2011 amplifica esse crescimento no contexto brasileiro, dado que determina que todo órgão e entidade pública ofereça o acesso às suas informações, mas não somente isso, se utilizando de procedimentos ágeis, de forma transparente, clara e em linguagem de fácil compreensão. A atuação governamental também vem crescendo em mídias sociais como um canal de comunicação e participação percebida pelos cidadãos (Song e Lee, 
2016). Contudo, tais informações públicas são de pouco proveito a menos que os cidadãos entendam o que elas significam. Em outros termos, possibilitar o acesso é apenas o primeiro passo para que uma informação alcance a transparência, por definição é necessário que a informação seja também entendida por quem a consome (Cappelli, 2009).

Algumas iniciativas favorecem esse entendimento como o uso de representações visuais e Linguagem Cidadã (Plain Language). Os recursos visuais pela naturalidade das suas formas em geral exigem menor esforço cognitivo à interpretação humana (Card et al., 1983). Já a Linguagem Cidadã busca apresentar, o texto, a estrutura e o design da informação tão claros que o usuário final consiga encontrar facilmente o que procura, compreender o que encontrou e usar essa informação (Plain Language Association International, 2021). Ela ajudará os cidadãos a entender o que lêem, podendo cumprir de modo mais fácil e rapidamente suas tarefas ou obter serviços perante o governo (Barboza, 2010). Ambas as técnicas podem ser combinadas para melhoria geral da comunicação multimídia atual. A Linguagem Cidadã se propõe a melhorias não apenas no âmbito textual, mas na estrutura e no design da informação. Identificamos que a mesma pode se aprofundar e oferecer também um método que abrange o tópico de visualização de dados. Sobretudo por não haver diretrizes para recursos visuais de forma específica no Federal Plain Language Guideline, conjunto oficial de orientações para traduzir uma informação para Linguagem Cidadã ${ }^{1}$. Neste guia apenas uma única diretriz se refere ao uso de imagens para clareza informacional, indicando apenas o uso ilustrativo. Apenas inserir imagens sem o cuidado necessário pode dificultar mais o processo de interpretação ou pouco contribuir na direção do entendimento da informação. Assim, o problema que este trabalho apresenta é: A falta de diretrizes específicas para visualização de informações na linguagem cidadã.

Desde os primórdios da visualização suportada por computadores, muitos especialistas buscam definir diretrizes, regras ou princípios para transferência do conhecimento de vários pontos do processo de criação de uma visualização. Apesar disso, não percebemos a existência de padrões ou normas de visualização, sobretudo no contexto público, para a inclusão à Linguagem Cidadã. A idealização de um conjunto padronizado de diretrizes pode apoiar a construção mais eficiente de visualizações tanto de forma manual como automatizada. Assim como favorecer a transparência onde estes recursos serão utilizados, facilitando também o entendimento do cidadão. $\mathrm{O}$ objetivo geral (OG) desta pesquisa é: Estender a linguagem cidadã através da inclusão de diretrizes específicas para projetar visualizações mais eficientes. Para isso, este trabalho possui como objetivos específicos:

- OE1: Revisar a literatura para captar as diretrizes existentes para melhoria do entendimento de informações em visualização;

- OE2: Construir um repositório único e centralizado de diretrizes para visualização de informação em Linguagem Cidadã;

Esta pesquisa inicial faz parte de um trabalho mais amplo e aqui estamos apresentando os resultados referentes ao OE1. Após esta introdução a Seção 2 apresenta o referencial teórico que fundamenta este artigo, posteriormente a metodologia que

\footnotetext{
${ }^{1}$ https://plainlanguage.gov/guidelines/
} 
norteia as etapas da pesquisa e o protocolo da revisão da literatura são descritas na Seção 3. Na Seção 4 formalizamos o conjunto de diretrizes para visualização e citamos exemplos, sendo esta a principal contribuição da pesquisa. Finalizando, na Seção 5 com a conclusão e trabalhos futuros.

\section{Referencial Teórico}

\subsection{Linguagem Cidadã}

O termo original Plain Language é de origem inglesa com suas variantes no português serem referenciadas desde linguagem clara, linguagem simples ou como preferido neste trabalho, linguagem cidadã. Com ações pelo Reino Unido ${ }^{2}$, Estados Unidos ${ }^{3}, \mathrm{Chile}^{4}$, e mais de 15 outros países, compõe uma série de técnicas, diretrizes e recomendações para apoiar uma escrita com mais clareza e objetividade. As aplicações surgem desde a simplificação da comunicação empresarial (Shumskaya, 2019) à vertentes para a gestão pública (Fischer et al., 2019). As instituições governamentais criam e disseminam grandes quantidades de informação e dados e, por isso, podem se aproveitar dessa técnica para melhoria dos seus serviços ${ }^{5}$. A linguagem cidadã é projetada para comunicar precisamente as informações para o público pretendido, com documentos claros e concisos (Barboza, 2010). Quanto à dinâmica textual, a linguagem cidadã oferece método para melhorar a inteligibilidade, entretanto no carácter visual é superficial. A rigor, não existe uma diretriz claramente definida sobre este tema. $\mathrm{O}$ uso para outras formas de apresentação da informação ainda é pouco discutido, evidenciando a contribuição desta pesquisa para ambas as áreas.

\subsection{Visualização de Informações}

A visualização de informações (Infovis) é uma subárea da visualização de dados e trata do processo de transformar informações que não são inerentemente espaciais em uma forma visual, geralmente com gráficos estatísticos em duas dimensões (Manovich, 2011). O seu objetivo principal é ajudar as pessoas a realizar tarefas com mais eficiência (Munzner, 2014) logo, são apresentadas como uma alternativa de solução para um maior alcance de entendimento dos dados públicos por parte dos cidadãos em geral. No entanto, não há foco em como essas visualizações devem ser construídas de maneira adequada para o consumo público (Park e Garcia, 2017). Quando o uso de diretrizes é necessário constatamos a falta de uma grande coleção delas que seja de domínio público e bem fundamentado (Engelke et al., 2018). Elas seguem citadas apenas em livros especializados, artigos ou em fóruns ${ }^{6}$. Oferecer uma Linguagem cidadã que contempla essas diretrizes aos desenvolvedores de visualização quer no contexto público ou geral, principalmente os não especialistas, pode facilitar e ampliar as adesões à transparência nestas instituições. Vale ressaltar que há trabalhos que se direcionam a sobrepor técnicas de linguagem cidadã e visualizações fora do contexto governamental, por exemplo, apresentando estratégias de contratos com escrita simples juntamente com visualizações para ofertar mais clareza aos serviços prestados (Passera, 2012). No entanto, apenas

\footnotetext{
${ }^{2}$ https://www.gov.uk/guidance/content-design/writing-for-gov-uk

${ }^{3} \mathrm{https}: / / \mathrm{www}$.plainlanguage.gov/

${ }^{4}$ http://www.lenguajeclarochile.cl/

${ }^{5} \mathrm{https} / / /$ www.gov.br/pt-br/guia-de-edicao-de-servicos-do-gov.br/escrevendo-para-o-seu-usuario

${ }^{6}$ https://visguides.org/
} 
representam soluções, não produzindo modelos e técnicas para outros contextos como a proposta deste trabalho o faz.

\section{Metodologia}

A metodologia 'Design Science Research' (DSR) guiará o desenvolvimento completo do artefato de pesquisa, a extensão à Linguagem Cidadã. No entanto, como este artigo inicial trata do objetivo específico OE1, destacamos a revisão da literatura e seus procedimentos a partir do trabalho de Kitchenham (2004) na subseção 3.1 a seguir.

\subsection{Revisão da Literatura}

O processo de revisão foi realizado seguindo as seguintes etapas (Figura 1): (i) Planejamento, com a definição dos objetivos, critérios de seleção, escolha da base de dados e formulação da string de busca; (ii) Condução, execução do que foi planejado em um estudo primário buscando artigos de referência, ajustes na string de busca, seleção e extração de dados nos artigos adquiridos segundo o protocolo; (iii) Documentação, validação por especialistas dos resultados obtidos e síntese dos dados obtidos.

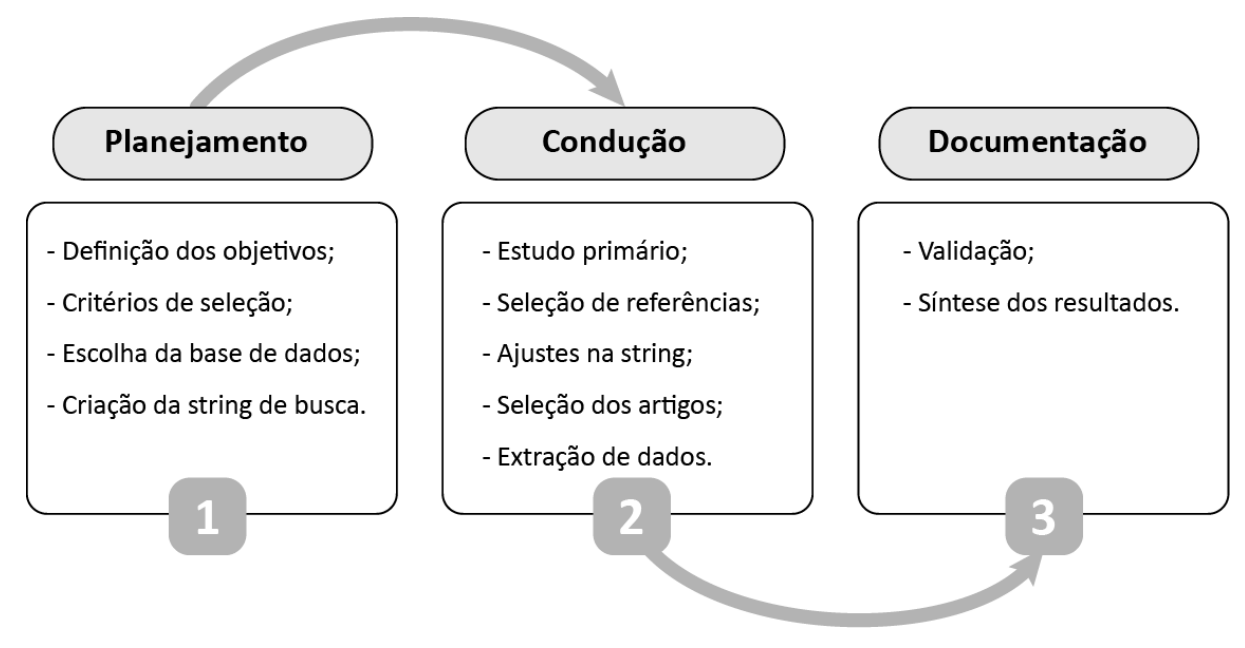

Figura 1. Etapas da revisão da literatura. Fonte. o autor.

No planejamento o objetivo estabelecido para a revisão foi de encontrar diretrizes, princípios, recomendações, boas práticas ou regras que tornem as visualizações mais eficientes, ou seja, corrijam erros, indiquem o que é mais assertivo e convencional na área de infovis. Os critérios de seleção foram: (i) $\mathrm{O}$ artigo é sobre os tipos de visualizações mais comuns (gráficos de mapas, linhas, barras, etc.)? Este critério foi incluído para limitar o escopo da pesquisa, uma vez que há inúmeras técnicas e tipos de gráficos de diversas complexidades. (ii) Descreve alguma diretriz sobre visualizações demonstradas com experimentos, 'expertise' de especialistas renomados ou de trabalhos clássicos na área? (iii) $\mathrm{O}$ acesso ao texto completo está disponível? (iv) Está escrito em inglês ou português? A biblioteca digital Scopus foi escolhida como a base de busca, uma vez que é maior banco de dados de resumos e citações da literatura com revisão por pares de revistas científicas, livros, processos de congressos e publicações ${ }^{7}$. Com base na estrutura PIO (population, interventions and outcomes)

\footnotetext{
${ }^{7}$ https://www.elsevier.com/pt-br/solutions/scopus
} 
(Kitchenham, 2004) e utilizando o Google Scholar na etapa inicial de formação de termos de buscas e artigos de referências foram recuperadas pesquisas a partir da string: TITLE-ABS-KEY ( ( visualization OR visualisation OR "visual perception" OR "visual representation" OR "visual encoding" OR "visual analys*") AND (rules OR recommendation OR principle OR technique OR "guide*" OR advice OR pattern OR "design practices" OR "good practice" ) AND ( "effec* visual*" OR "efficient visual* " ) ).

A extração de dados em junho de 2020, retornou 976 artigos. O número expressivo resultante é devido às várias expressões e sinônimos. Estes tornaram os resultados mais amplos. Ao se restringir alguns deles, artigos de referências não eram contemplados, reforçando a falta de padronização dos termos da área. Na primeira filtragem de leitura do título e resumo permaneceram 50 trabalhos para leitura completa. Numa segunda triagem foram descartados os trabalhos distantes do objetivo da revisão e do escopo delimitado, resultando em 9 artigos finais (Lee N; Rojas, 2009), (Isett; Hicks, 2018), (Senay et al., 1990), (Kelleher; Wagener, 2011), (Rheingans, 2000), (Siirtola, 2019), (Grainger et al., 2016), (Koop et al., 2018), (Gramazio et., 2014). De forma a enriquecer nosso repositório com diretrizes de especialistas foram selecionados alguns livros com base no trabalho de Rees e Laramee (2019) que fornecem indicações das obras mais relevantes para cada tópico da área. Os livros escolhidos e analisados integralmente refletem essas categorias como Munzner (2014) sendo a referência acadêmica e profissionais do setor com Knaflic (2015). Adicionalmente o livro de Wilke (2019) foi o título mais recente inserido para incorporar os princípios mais atuais da área. Dispensamos obras das categorias especializadas e ferramentas por tratarem de assuntos específicos, visto que nosso trabalho reflete diretrizes mais generalizáveis.

\section{Extensão da Linguagem Cidadã para Visualização de Informações}

Ao todo 157 diretrizes foram incluídas (86 dos livros e 71 da revisão da literatura). Todas as diretrizes foram sintetizadas por similaridade ou por abranger o mesmo princípio como: (i) Apresentação, cuidados acerca do contexto; (ii) Canal Visual, dimensões que a informação é apresentada; (iii) Cor, não se trata da cor como elemento estético, mas como um canal visual; (iv) Design, reflete os cuidados essenciais com recursos que não são dos dados em si mais da forma de apresentá-los esteticamente adequados; (v) Eixos, acerca dos rótulos específicos das variáveis; (vi) Marcas visuais, trata dos tipos de símbolos e suas relações. Ao todo 55 diretrizes formam essa extensão da linguagem cidadã para visualizações, divididas pelas categorias acima e mais algumas específicas apenas dos tipos de gráficos mais recorrentes (barras, linhas, pizza ou mapas) de acordo com o escopo da pesquisa. As diretrizes selecionadas não visam representar todas as boas práticas da área de forma completa, mas formar um conjunto inicial mínimo que ofereça um design mais eficiente. Nenhum julgamento de valor foi feito sobre as diretrizes, presume-se que todas são de igual importância. A ausência de qualquer uma delas é considerada uma falha, prejudicando a compreensão do gráfico em algum aspecto ou deixando de torná-lo mais transparente ou mais fácil de ler possível. Na Tabela 1 compilamos uma amostra dessas diretrizes, por motivos de limitação de páginas o conjunto integral dessas regras não foi adicionado, mas ele está disponível em: https://github.com/RodOlive/Indicator_Vizualization_Ineficient. 
Tabela 1. Diretrizes para visualizações eficientes.

\begin{tabular}{|c|c|c|}
\hline Tipo & Diretriz & Referências \\
\hline $\begin{array}{l}\text { Canal } \\
\text { visual }\end{array}$ & $\begin{array}{l}\text { Adicione canais visuais redundantes do mesmo dado para } \\
\text { facilitar a percepção. }\end{array}$ & $\begin{array}{l}\text { (Munzner, 2014. P.97-98), (Wilke, } \\
\text { 2019. P.243-246), (Rheingans, 2000) }\end{array}$ \\
\hline Cor & $\begin{array}{l}\text { A diferença de tons de uma mesma cor deve ser evidente } \\
\text { em qualquer escala de cores }\end{array}$ & (Wilke, 2019. P.30-32) \\
\hline Cor & Use os significados culturais das cores a seu favor. & $\begin{array}{l}\text { (Rheingans, 2000), (Grainger et al., } \\
\text { 2016) }\end{array}$ \\
\hline Design & Não use 3D sem justificativa. & $\begin{array}{l}\text { (Munzner, 2014. P.117,118), (Wilke, } \\
\text { 2019. P.305-307) }\end{array}$ \\
\hline Design & $\begin{array}{l}\text { Não mostre muitos dados de uma só vez ou informações } \\
\text { desnecessárias. Isso gera desordem visual }\end{array}$ & $\begin{array}{l}\text { (Isett e Hicks, 2018), (Wilke, } 2019 . \\
\text { P.337-340), (Grainger et al., 2016), } \\
\text { (Lee N e Rojas, 2009) }\end{array}$ \\
\hline Eixos & Mantenha sempre um título sobre todo o gráfico & $\begin{array}{l}\text { (Isett; Hicks, 2018), (Senay e } \\
\text { Ignatius, 1994), (Wilke, } 2019 . \\
\text { P.267-268) }\end{array}$ \\
\hline Eixos & Rotule cada eixo com títulos e suas unidades & $\begin{array}{l}\text { (Senay e lgnatius, 1994), (Wilke, } \\
\text { 2019. P.270-273), (Isett e Hicks, } \\
\text { 2018) }\end{array}$ \\
\hline $\begin{array}{l}\text { Marca } \\
\text { Visual }\end{array}$ & $\begin{array}{l}\text { Não repita a mesma marca visual para dados diferentes, } \\
\text { para um item distinto se destacar de muitos outros } \\
\text { imediatamente. }\end{array}$ & $\begin{array}{l}\text { (Wilke, 2019. P.10-12), (Isett e Hicks, } \\
\text { 2018), (Senay e lgnatius, 1994), } \\
\text { (Munzner, 2014. P.109-111) }\end{array}$ \\
\hline Legenda & $\begin{array}{l}\text { Projete a figura de tal maneira que seja óbvio o que os } \\
\text { vários elementos gráficos representam. Elimine legendas } \\
\text { sempre que possível. }\end{array}$ & (Wilke, 2019. P.250-253) \\
\hline Barras & $\begin{array}{l}\text { Inverta os eixos ao invés de rotacionar os rótulos de eixo } \\
\text { grandes. }\end{array}$ & (Wilke, 2019. P.45-48) \\
\hline Pizza & $\begin{array}{l}\text { Enfatize frações simples e não deixe fatias pequenas. Isso } \\
\text { dificulta a comparação. }\end{array}$ & $\begin{array}{l}\text { (Siirtola, 2019), (Wilke, } 2019 . \\
\text { P.97-99) }\end{array}$ \\
\hline Mapas & Adicione somente camadas de informações relevantes. & (Wilke, 2019. P.169) \\
\hline Linha & $\begin{array}{l}\text { Use a largura da linha para diferenciar até no máximo } \\
\text { quatro valores. }\end{array}$ & (Munzner, 2014. P.106,107) \\
\hline
\end{tabular}

\section{Conclusões}

Este trabalho tem como contribuição principal a criação de um conjunto de diretrizes de visualização de informações, com base na revisão da literatura. Nossa intenção é que estas possam ser uma extensão do Guia de práticas da Linguagem Cidadã. As próximas etapas serão: (i) Busca em outras bases de dados como Web of Science, ACM Library, IEEE, entre outras para obter a saturação teórica em diretrizes, além da realização de uma avaliação junto a especialistas para acréscimo de recomendações do estado da prática; (ii) Elaborar um guia didático com as diretrizes compiladas de forma a auxiliar o desenvolvedor a pôr em prática tais conceitos; (iii) Avaliar a facilidade de uso e aceitação dessas diretrizes em um estudo de caso; (iv) Integrar a esta solução mais regras de outros tipos de gráficos mais complexos e também diretrizes para outros aspectos de visualização, como interatividade, que também atuam como um aliados na compreensão dos dados. Por fim, será desenvolvido um verificador automatizado que, ao detectar violações dessas diretrizes, ajudará o usuário final não especialista a 
construir uma visualização mais inteligível. Esse verificador poderá ser acoplado ao software de design de visualização disponível ou em modelos de inteligência artificial para a construção desses artefatos.

\section{Referências}

Barcellos, R. Avaliação da Qualidade e Interpretabilidade de Visualizações de Dados. (Dissertação de Mestrado, Universidade Federal Fluminense) Niterói, 2017. 87 p.

Brasil. (2011) Lei $\mathrm{n}^{\mathrm{o}} 12.527$ - Lei de acesso à informação. Disponível em: $<$ http://www.planalto.gov.br/ccivil 03/ ato2011-2014/2011/lei/112527.htm> Acesso em 01 de junho de 2021.

Song, C., \& Lee, J. (2016). Citizens' use of social media in government, perceived transparency, and trust in government. Public Performance \& Management Review, $39(2), 430-453$.

Cappelli, C. Uma Abordagem para Transparência em Processos Organizacionais Utilizando Aspectos. Tese de Doutorado, PUC-Rio, Rio de Janeiro, Brasil, 2009.

Card, S. K.; Moran, T. P.; Newell, A. The Psychology of Human-Computer Interaction. Lawrence Erlbaum Associates, New Jersey, 1983.

Plain Language Association International. What is plain language? 2021. Disponível em: <https://plainlanguagenetwork.org/plain-language/what-is-plain-language/> Acesso em 15 de maio de 2021.

Barboza, E. M. F. (2010). A linguagem clara em conteúdos de websites governamentais para promover a acessibilidade a cidadãos com baixo nível de escolaridade. Inc. Soc., Brasília, DF, v. 4 n. 1, p.52-66, jul./dez. 2010.

Shneiderman, B. (1996). The eyes have it: A task by data type taxonomy for information visualizations. In Proceedings 1996 IEEE symposium on visual languages (pp. 336-343). IEEE.

Fischer, H., Mont'Alvão, C., \& dos Santos Rodrigues, E. (2019). O Papel do Texto na Compreensibilidade de E-serviços. Ergodesign \& HCI, 7(Especial), 207-219.

Barboza, E. M. F. (2010). A linguagem clara em conteúdos de websites governamentais para promover a acessibilidade a cidadãos com baixo nível de escolaridade. Inc. Soc., Brasília, DF, v. 4 n. 1, p.52-66, jul./dez. 2010

Munzner, Tamara. Visualization analysis and design. CRC press, 2014.

Park, S., \& Gil-Garcia, J. R. (2017). Understanding transparency and accountability in open government ecosystems: The case of health data visualizations in a state government. In Proceedings of the 18th Annual International Conference on Digital Government Research (pp. 39-47).

Engelke, U., Abdul-Rahman, A., \& Chen, M. (2018). VISupply: A Supply-Chain Process Model for Visualization Guidelines. In 2018 International Symposium on Big Data Visual and Immersive Analytics (BDVA) (pp. 1-9). IEEE. 
Passera, S. (2012). Enhancing contract usability and user experience through visualization-an experimental evaluation. In 2012 16th International Conference on Information Visualisation (pp. 376-382). IEEE.

Kitchenham, B. (2004). Procedures for performing systematic reviews. Keele, UK, Keele University, 33(2004), 1-26.

Lee, N., \& Rojas, E. M. (2009). Developing effective visual representations to monitor project performance. In Construction Research Congress 2009: Building a Sustainable Future (pp. 826-835).

Isett, K. R., \& Hicks, D. M. (2018). Providing public servants what they need: Revealing the "unseen" through data visualization. Public Administration Review, 78(3), 479-485.

Senay, H., \& Ignatius, E. (1990). Rules and principles of scientific data visualization. Institute for Information Science and Technology, Department of Electrical Engineering and Computer Science, School of Engineering and Applied Science, George Washington University.

Kelleher, C., \& Wagener, T. (2011). Ten guidelines for effective data visualization in scientific publications. Environmental Modelling \& Software, 26(6), 822-827.

Rheingans, P. L. (2000). Task-based color scale design. In 28th AIPR Workshop: 3D Visualization for Data Exploration and Decision Making (Vol. 3905, pp. 35-43). International Society for Optics and Photonics.

Siirtola, H. (2019). The cost of pie charts. In 2019 23rd International Conference Information Visualisation (IV) (pp. 151-156). IEEE.

Grainger, S., Mao, F., \& Buytaert, W. (2016). Environmental data visualisation for non-scientific contexts: Literature review and design framework. Environmental Modelling \& Software, 85, 299-318.

Kopp, T., Riekert, M., \& Utz, S. (2018). When cognitive fit outweighs cognitive load: Redundant data labels in charts increase accuracy and speed of information extraction. Computers in Human Behavior, 86, 367-376.

Gramazio, C. C., Schloss, K. B., \& Laidlaw, D. H. (2014). The relation between visualization size, grouping, and user performance. IEEE transactions on visualization and computer graphics, 20(12), 1953-1962.

Rees, D., \& Laramee, R. S. (2019). A survey of information visualization books. In Computer Graphics Forum (Vol. 38, No. 1, pp. 610-646).

Knaflic, C. N. (2015). Storytelling with data: A data visualization guide for business professionals. John Wiley \& Sons.

Wilke, C. O. (2019). Fundamentals of data visualization: a primer on making informative and compelling figures. O'Reilly Media. 